\title{
PRODUCTS OF CW-COMPLEXES
}

\author{
YOSHIO TANAKA
}

ABSTRACT. We show that Liu's characterization for the product $K \times L$ to be a $\mathrm{CW}$-complex is independent of the usual axioms of set theory.

1. Introduction. The concept of CW-complex due to J. H. C. Whitehead [8] is well known. Recall that a space $K$ is a $C W$-complex, if it is a complex with cells $\left\{e_{\alpha}\right\}$ such that each $e_{\alpha}$ is contained in a finite subcomplex, and $K$ has the weak topology with respect to a closed cover $\left\{\bar{e}_{\alpha}\right\}$; that is, $F \subset K$ is closed in $K$ if $F \cap \bar{e}_{\alpha}$ is closed for each $\bar{e}_{\alpha}$. Every Whitehead complex introduced by C. H. Dowker [1] is a CW-complex with cells $\left\{e_{\alpha}\right\}$ such that each $\bar{e}_{\alpha}$ is a subcomplex.

Let $K$ be a CW-complex with cells $\left\{e_{\alpha}\right\}$. Then $K$ is called locally finite; locally countable, if for each $x \in K$, there is respectively a finite; countable subcomplex $A$ of $K$ with $x \in$ int $A$. Hence $K$ is locally finite; locally countable, if and only if a closed cover $\left\{\bar{e}_{\alpha}\right\}$ of the space $K$ is so respectively.

Liu Ying-ming [3], assuming the continuum hypothesis $(\mathrm{CH})$, gave the following necessary and sufficient condition for the product of two $\mathrm{CW}$-complexes to be a CW-complex.

$(\mathrm{CH})$. Let $K$ and $L$ be $\mathrm{CW}$-complexes. Then $K \times L$ is a CW-complex if and only if $K$ or $L$ is locally finite, or $K$ and $L$ are locally countable.

On the other hand, assuming $(\mathrm{CH})$, we gave a necessary and sufficient condition for the product of two closed images of metric spaces to be a $k$-space [5]. G. Gruenhage [2] showed that this characterization is equivalent to a certain set-theoretic axiom weaker than $(\mathrm{CH})$.

In this paper, analogously we shall show that Liu's result is in fact equivalent to this set-theoretic axiom. And also, if $K=L$, this result is valid without any set-theory beyond ZFC. These are affirmative answers to the questions in [6]. Many of the results in this paper were also obtained by Zhou Hao-xuan in his paper [9]. The author wishes to thank him for his translation of Liu's paper [3].

2. Results. First of all, we shall recall the well-known examples below. Let $S_{\omega}$ be a sequential fan; that is, it is the quotient space obtained from the topological sum of $\omega$ convergent sequences by identifying all the limit points. $S_{\alpha}, \alpha>\omega$, are similarly defined replacing " $\omega$ " by " $\alpha$ ". We also need another canonical example $S_{2}$. That is, $S_{2}$ is the space $(N \times N) \cup N \cup\{0\}$ with each point of $N \times N$ isolated, $N$ is the set

Received by the editors July 13, 1981 and, in revised form, January 5, 1982 1980 Mathematics Subject Classification. Primary 54E60; Secondary 54B10. Key words and phrases. CW-complex, $k$-space, $B F(\alpha)$. 
of natural numbers. A basis of neighborhoods of $n \in N$ consists of all sets of the form $\{n\} \cup\left\{(m, n): m \geqslant m_{0}\right\}$. And $U$ is a neighborhood of 0 if and only if $0 \in U$ and $U$ is a neighborhood of all but finitely many $n \in N$. We remark that $S_{\omega}$ is the perfect image of $S_{2}$ by identifying all points of a compact subst $N \cup\{0\}$ of $S_{2}$. This will be used later.

In [7], we showed that the metrizability of certain quotient images of metric spaces can be characterized by whether or not they contain copies of $S_{\omega}$ and $S_{2}$. As for $\mathrm{CW}$-complexes, we have the following by invoking [6, Proposition 2.3] and the well-known fact that every $\mathrm{CW}$-complex is locally finite if and only if it is metrizable.

LEMMA 1. Let $K$ be a $\mathrm{CW}$-complex. Then the following are equivalent.

(1) $K$ is metrizable.

(2) $K$ is locally finite.

(3) $K$ contains no closed copy of $S_{\omega}$ and no $S_{2}$.

Now, we consider the products of $\mathrm{CW}$-complexes in terms of a certain set-theoretic axiom weaker than $(\mathrm{CH})$.

Let ${ }^{N} N$ be the set of all functions from $N$ into $N$. For $f, g \in{ }^{N} N$, we define $f \leqslant g$ if and only if $\{n \in N ; f(n)>g(n)\}$ is finite. For infinite cardinal $\alpha$, by $B F(\alpha)$ we mean the following assertion:

$B F(\alpha)$ : If $F \subset \subset^{N} N$ has cardinality less than $\alpha$, then there exists $g \in{ }^{N} N$ such that $f \leqslant g$ for all $f \in F$.

It is well known that Martin's Axiom implies that $B F(\alpha)$ holds for all $\alpha$ less than or equal to the continuum. It is easy to show that $(\mathrm{CH})$ implies that $B F\left(\omega_{2}\right)$ is false. As for $B F(\alpha), G$. Gruenhage [2] gave the following equivalence in terms of products of $k$-spaces, $\alpha^{+}$denotes the least cardinal greater than $\alpha$. Recall that a space $X$ is a $k$-space if it has the weak topology with respect to the cover consisting of all compact subsets of $X$.

(*) $B F\left(\alpha^{+}\right)$holds if and only if $S_{\omega} \times S_{\alpha}$ is a $k$-space.

To apply this result $(*)$ to the products of $\mathrm{CW}$-complexes, let $I_{\alpha}$ be the quotient space obtained from the topological sum of $\alpha$ closed unit intervals $[0,1]$ by identifying all the zero points. Then the result $(*)$ suggests the following.

LEMMA 2. $B F\left(\alpha^{+}\right)$holds if and only if $I_{\omega} \times I_{\alpha}$ is a CW-complex.

Proof. If $B F\left(\alpha^{+}\right)$holds, then it follows from the proof of [2, Lemma 1] that $I_{\omega} \times I_{\alpha}$ is sequential, hence a CW-complex by [6, Proposition 2.5]. If $I_{\omega} \times I_{\alpha}$ is a $\mathrm{CW}$-complex, hence a $k$-space, then $S_{\omega} \times S_{\alpha}$ is a k-space, for $S_{\omega} \times S_{\alpha}$ is closed in $I_{\omega} \times I_{\alpha}$. Hence, by the result $(*), B F\left(\alpha^{+}\right)$holds.

Lemma 3. Suppose that $e$ is a cell of a CW-complex $K$ such that $x \in e$ and each neighborhood of $x$ in e meets at least $\omega_{1}$ many boundaries of cells of $K$. Suppose that $L$ is a CW-complex which is not locally finite. Then $K \times L$ is not a $\mathrm{CW}$-complex if $B F\left(\omega_{2}\right)$ is false. 
Proof. Since $e$ is first countable, there is a decreasing local base $\left\{G_{n} ; n \in N\right\}$ of $x$ in $e$. By the hypothesis, there exist pairwise disjoint collections $\Omega_{n}, n \in N$, of cells of $K$ such that $\left|\Omega_{n}\right|=\omega_{1}$, and boundary of each cell of $\Omega_{n}$ meets $G_{n}$. For each $e \in \Omega_{n}$, let $x(e) \in \partial e \cap G_{n}$, and let $\left\{S_{n}(e) ; n \in N\right\}$ be a decreasing local base of $x(e)$ in $\bar{e}$. Then there exists a subset $\{x(e, n) ; n \in N\}$ of $e$ with $x(e, n) \in S_{n}(e)$. Now, since the CW-complex $L$ is not locally finite, by Lemma $1, L$ contains a closed copy of $S_{\omega}$ or $S_{2}$. Suppose that $K \times L$ is a CW-complex, hence a $k$-space. If $L$ contains a closed copy of $S_{\omega}$, then $K \times S_{\omega}$ is a $k$-space. If $L$ contains a closed copy $S_{2}$, then $K \times S_{2}$ is a $k$-space. But, $S_{\omega}$ is the perfect image of $S_{2}$, so $K \times S_{\omega}$ is the perfect image of $K \times S_{2}$. Thus $K \times S_{\omega}$ is a $k$-space. Hence, in any case, $K \times S_{\omega}$ is a $k$-space.

Now, since $B F\left(\omega_{2}\right)$ is false, there is a collection $\left\{f_{\alpha} ; f_{\alpha}: N \rightarrow N, \alpha<\omega_{1}\right\}$ such that if $f: N \rightarrow N$, then there exists $\alpha<\omega_{1}$ with $f_{\alpha}(n)>f(n)$ for infinitely many $n \in N$. Since each $\Omega_{n}$ has cardinality of $\omega_{1}$, we can put $\Omega_{n}=\left\{e_{\alpha}^{n} ; \alpha<\omega_{1}\right\}$. For each $j \in N$, let

$$
H_{j}=\bigcup_{\alpha \in \omega_{1}}\left\{\left(x\left(e_{\alpha}^{j}, n\right),(n, m)\right) ; f_{\alpha}(n) \geqslant m\right\},
$$

and $H=\cup_{j \in N} H_{j}$, where $(n, m)$ is the $m$ th term of the $n$th sequence in $S_{\omega}$. Then it is easy to show that $H \cap C$ is finite for each compact subset $C$ of $K \times S_{\omega}$, because each compact subset of $K\left(S_{\omega}\right)$ meets only finitely many cells of $K$ (sequences in $S_{\omega}$ ). Since $K \times S_{\omega}$ is a $k$-space, $H$ is closed in $K \times S_{\omega}$. We obtain a contradiction by showing that $(x, \infty) \in \bar{H}$, where $\infty$ is the nonisolated point in $S_{\omega}$. This contradiction implies that $K \times L$ is not a CW-complex. Thus it remains to show that $(x, \infty) \in \bar{H}$. Let $W=U \times V$ be a neighborhood of $(x, \infty)$ in $K \times S_{\omega}$, and let $G_{l} \subset U$. For each $n \in N$, since $V$ is a neighborhood of $\infty$ in $S_{\omega}$, there exists $n^{\prime} \in N$ such that $n^{\prime}>n$ and $(n, m) \in V$ if $m \geqslant n^{\prime}$. Let $f: N \rightarrow N$ be defined by $f(n)=n^{\prime}$. Then there exists $\alpha_{0}<\omega_{1}$ such that $f_{\alpha_{0}}(n)>f(n)$ for infinitely many $n \in N$. Since $x\left(e_{\alpha_{0}}^{l}, n\right) \rightarrow x\left(e_{\alpha_{0}}^{l}\right)$ $\in U$, and $U$ is open in $K$, there exists $n_{0} \in N$ with $x\left(e_{\alpha_{0}}^{l}, n_{0}\right) \in U$ and $f_{\alpha_{0}}\left(n_{0}\right)>$ $f\left(n_{0}\right)$. Then, $\left(x\left(e_{\alpha_{0}}^{l}, n_{0}\right),\left(n_{0}, f_{\alpha_{0}}\left(n_{0}\right)\right)\right) \in H_{l} \cap(U \times V)$. This implies that $(x, \infty) \in$ $\bar{H}$.

LemMa 4 [3, LemMa 3]. Let $K$ be a CW-complex. Suppose that for $x \in K$ and a cell $e$ of $K$ with $x \in e$, there is a neighborhood $U$ of $x$ in $e$ such that $\mid\{e \in K$; $\partial e \cap U \neq \varnothing\} \mid \leqslant \omega$. Then $K$ is locally countable at $x$.

Now we are ready for the main result concerning products.

THEOREM 5. Let $K$ and $L$ be $\mathrm{CW}$-complexes. Then the following are equivalent.

(1) $B F\left(\omega_{2}\right)$ is false.

(2) $K \times L$ is a CW-complex if and only if $K$ or $L$ is locally finite, otherwise $K$ and $L$ are locally countable.

Proof. (2) $\rightarrow(1)$ : Suppose that $B F\left(\omega_{2}\right)$ holds. Then, by Lemma $2, I_{\omega} \times I_{\omega_{1}}$ is a $\mathrm{CW}$-complex. But, in this case, the "only if" part does not hold. Hence $B F\left(\omega_{2}\right)$ is false. 
(1) $\rightarrow$ (2): The "if" part of (2) is well known. Indeed, it essentially follows from results of $[8,(\mathrm{H})]$ and $[4$, Lemma 2.1]. So we prove the "only if" part. Suppose that $K$ is not locally countable and also $L$ is not locally finite. Since $K$ is not locally countable, by Lemma 4 there is $x \in e$ such that every neighborhood $x$ in $e$ meets at least $\omega_{1}$ many boundaries of cells of $K$. Thus, since $B F\left(\omega_{2}\right)$ is false, $K \times L$ is not a $\mathrm{CW}$-complex by Lemma 3. This is a contradiction. Hence $L$ is locally finite if $K$ is not locally countable. Similarly, $K$ is locally finite if $L$ is not locally countable. That completes the proof.

Lemma 6. Suppose that $e ; \tau$ is respectively a cell of a $\mathrm{CW}$-complex $K ; L$ such that $x \in e ; y \in \tau$, and each neighborhood of $x$ in $e ; y$ in $\tau$ meets at least $\omega_{1}$ many boundaries of cells of $K$; L. Then $K \times L$ is not a $\mathrm{CW}$-complex.

Proof. For each $\alpha<\omega_{1}$, let $f_{\alpha}: \omega_{1} \rightarrow N$ be a function such that $f_{\alpha}$ restricted to $\alpha$ is a one-to-one map onto $N$. Let $\left\{G_{n} ; n \in N\right\}$, and $\left\{x\left(e_{\alpha}^{j}, n\right) ; j, n \in N, \alpha<\omega_{1}\right\}$ be the same as in the proof of Lemma 3. Similarly define $\left\{G_{n}^{\prime}: n \in N\right\}$ and $\left\{y\left(\tau_{\alpha}^{j}, n\right)\right.$; $\left.j, n \in N, \alpha<\omega_{1}\right\}$ in $L$. For each $j \in N$, let $M_{j}=\cup_{\alpha, \beta<\omega_{1}}\left\{\left(x\left(e_{\alpha}^{j}, n\right), y\left(\tau_{\beta}^{j}, f_{\beta}(\alpha)\right)\right)\right.$; $\left.n<f_{\beta}(\alpha)\right\}$, and $M=\cup_{j \in N} M_{j}$. Let us now suppose that $K \times L$ is a CW-complex, hence a $k$-space. Then $M$ is closed in $K \times L$, because $M \cap C$ is finite for each compact subset $C$ of $K \times L$. However, we have a contradiction that $(x, y) \in \bar{M}-M$ by referring to the proof of [2, Lemma 5], hence $K \times L$ is not a CW-complex. Indeed, to show $(x, y) \in \bar{M}$, let $U \times V$ be a neighborhood of $(x, y)$ in $K \times L$, and $G_{l} \subset U, G_{l}^{\prime} \subset V$. Then there is a function $g: \omega_{1} \rightarrow N$ such that for each $\alpha<\omega_{1}$, $\left\{x\left(e_{\alpha}^{l}, n\right) ; n \geqslant g(\alpha)\right\} \subset U$ and $\left\{y\left(\tau_{\alpha}^{l}, n\right) ; n \geqslant g(\alpha)\right\} \subset V$. Thus there is $n_{0} \in N$ and an uncountable subset $A$ of $\omega_{1}$ with $g(\alpha)=n_{0}$ if $\alpha \in A$. Let $\gamma$ be an element of $A$ which has infinitely many predecessors in $A$. Hence there is $\delta \in A$ with $\delta<\gamma$ and $f_{\gamma}(\delta)=m>n_{0}$. Thus $\left(x\left(e_{\delta}^{l}, n_{0}\right), y\left(\tau_{\gamma}^{l}, f_{\gamma}(\delta)\right)\right) \in M \cap(U \times V)$. Hence $M \cap(U \times$ $V) \neq \varnothing$, which implies $(x, y) \in \bar{M}$.

By the previous lemma and Lemma 4 , we have

THEOREM 7. If $K \times L$ is a CW-complex, then $K$ or $L$ is locally countable. When $K=L$, the converse holds.

RemARK. Let $K$ be a CW-complex. For each infinite cardinal $\alpha$, let us call $K$ locally $\alpha$, if $\alpha=\min \{\gamma$, for any $x \in K$, there is a subcomplex $A$ consisting of $\leqslant \gamma$ many cells with $x \in$ int $A\}$ (hence, $\alpha=\min \{\gamma$; for any $x \in K$, there is a neighborhood of $x$ which meets $\leqslant \gamma$ many closed cells $\bar{e}\}$ ).

Then we have the following analogue to Theorem 5 by Theorem 7, Lemma 2, and slight modifications of Lemmas 3 and 4 (cf. [9, Theorem 2.7]).

THEOREM. $B F\left(\alpha^{+}\right)$is false if and only if whenever $K \times L$ is $\mathrm{CW}$-complex, $K$ or $L$ is locally finite, otherwise one of $K, L$ is locally countable and another is locally $<\alpha$. 


\section{REFERENCES}

1. C. H. Dowker, Topology of metric complexes, Amer. J. Math. 74 (1952), 557-577.

2. G. Gruenhage, $K$-spaces and products of closed images of metric spaces, Proc. Amer. Math. Soc. 80 (1980), 478-482.

3. Liu Ying-ming, A necessary and sufficient condition for the products of $\mathrm{CW}$-complexes. Acta Math. Sinica 21 (1978), 171-175. (Chinese)

4. J. Milnor, Construction of universal bundles. I, Ann. of Math. 63 (1956), 272-284.

5. Y. Tanaka, $A$ characterization for the product of closed images of metric spaces to be a $k$-space. Proc. Amer. Math. Soc. 74 (1979), 166-170.

6. __ Products of spaces of countable tightness, Topology Proc. 6 (1981), 115-133.

7.

8. J. H. C. Whitehead, Combinatorial homotopy: I, Bull. Amer. Math. Soc. 55 (1949), 213-245.

9. Zhou Hao-xuan, Weak topology and J. Whitehead's problem (preprint).

Department of Mathematics, Tokyo Gakugei University, 4-1-1 Nukuikita-machi, Koganei-Shi, TOKYO, JAPAN 